\title{
Volterra Series Digital Backpropagation Accounting for PMD
}

\author{
Cristian B. Czegledi(1,2) and Ronen $\operatorname{Dar}^{(1)}$
}

(1) Nokia Bell Labs, 791 Holmdel Road, Holmdel, NJ 07733, USA

(2) Dept. of Electrical Engineering, Chalmers University of Technology, Gothenburg, SE-412 96, Sweden

\begin{abstract}
We propose a modified Volterra series digital backpropagation algorithm that accounts for polarization-mode dispersion (PMD) effects. The algorithm accounts only for nonlinear terms that are PMD-insensitive, leading to both performance enhancement and substantial complexity reduction.
\end{abstract}

\section{Introduction}

Nonlinear distortions caused by the inherent intensity-dependent Kerr nonlinearity of optical fibers represent a severe bottleneck on the achievable data rates of optical communication systems. Digital backpropagation (DBP) is a universal technique used to compensate for such nonlinear distortions. It involves digitally solving the nonlinear Schrödinger equation using the split-step Fourier method (SSFM) ${ }^{1}$ or using perturbation analysis in the time domain ${ }^{2}$ or frequency domain ${ }^{3}$. The latter approach is often referred to as Volterra series (VS) ${ }^{4}$ DBP.

Originally, both the SSFM and the perturbationbased DBP algorithms have been proposed without accounting for the effects of polarization-mode dispersion (PMD). The stochastic nature of PMD affects the way in which nonlinearities accumulate during the forward propagation, impairing the accuracy of the backward propagation and limiting the achievable DBP gains ${ }^{5}$. Recently, several works have showed that SSMF-DBP algorithms perform better when the accumulated PMD is accounted for in a distributed manner during the backward propagation rather than being completely removed before or after the DBP procedure ${ }^{6,7}$.

In this work we propose a modified VS-DBP algorithm that accounts for PMD by distinguishing between nonlinear contributions that are sensitive and insensitive to PMD. As opposed to conventional DBP algorithms, which ignore the effect of PMD and employ full-bandwidth backpropagation, our method acts like a sliding window that scans the entire bandwidth of the backpropagated signal, ignoring PMD-sensitive contributions (which may have a detrimental effect on the effectiveness of DBP) and properly accounting for the ones that are PMD insensitive. We show that our approach provides higher achievable gains compared to conventional DBP algorithms at substantially reduced computational effort.

\section{Theoretical background}

We consider dual-polarization single-channel transmission over a fiber link of length $L$. The spectral component of the received signal at angular frequency $\omega$, in the absence of amplified spontaneous emission (ASE) noise, can be written as $\mathbf{r}(\omega)=\mathbf{a}(\omega)+\Delta \mathbf{a}(\omega)$, where the two-element column vectors $\mathbf{a}(\omega)$ and $\Delta \mathbf{a}(\omega)$ represent the transmitted signal and the accumulated nonlinear interference in the two polarizations, assuming chromatic dispersion (CD) and PMD removal. Based on a first-order perturbation analysis, the term $\Delta \mathbf{a}(\omega)$ is given by

$$
\Delta \mathbf{a}(\omega)=\iint \boldsymbol{\psi}\left(\omega_{1}, \omega_{2}, \omega_{3}\right) \mathrm{d} \omega_{1} \mathrm{~d} \omega_{2},
$$

where $\omega_{3}=\omega-\omega_{1}+\omega_{2}$. The term $\boldsymbol{\psi}\left(\omega_{1}, \omega_{2}, \omega_{3}\right)$ represents the four-wave mixing (FWM) generated by the spectral components of the optical signal at angular frequencies $\omega_{1}, \omega_{2}$, and $\omega_{3}$, and it is given by

$$
\begin{gathered}
\boldsymbol{\psi}\left(\omega_{1}, \omega_{2}, \omega_{3}\right)=\int_{0}^{L} \rho\left(\omega_{1}, \omega_{2}, \omega_{3}, z\right) \mathbf{J}(\omega, z)^{\dagger} \mathbf{J}\left(\omega_{3}, z\right) \\
\times \mathbf{a}\left(\omega_{3}\right) \mathbf{a}\left(\omega_{2}\right)^{\dagger} \mathbf{J}\left(\omega_{2}, z\right)^{\dagger} \mathbf{J}\left(\omega_{1}, z\right) \mathbf{a}\left(\omega_{1}\right) \mathrm{d} z,
\end{gathered}
$$

where $\rho\left(\omega_{1}, \omega_{2}, \omega_{3}, z\right)=i \gamma \frac{8}{9} f(z) \exp \left(i \beta_{2}\left(\omega_{3}-\right.\right.$ $\left.\left.\omega_{2}\right)\left(\omega_{2}-\omega_{1}\right) z\right)$ with $f(z), \gamma$, and $\beta_{2}$ accounting for the power profile, nonlinear coefficient, and dispersion coefficient of the fiber. The $2 \times 2$ matrix $\mathbf{J}(\omega, z)$ models the accumulated polarization rotation at angular frequency $\omega$ and distance $z$.

In transmission scenarios with no PMD the polarization rotation matrix $\mathbf{J}(\omega, z)$ is fixed for all angular frequencies $\omega$, meaning that $\mathbf{J}(\omega, z)^{\dagger} \mathbf{J}(\omega+\Delta \omega, z)$ is equal to the identity matrix for any $\Delta \omega$, and all FWM terms in Eq. (1) are independent of PMD. In transmission scenarios with PMD the polarization rotation matrices at two different frequencies $\mathbf{J}(\omega, z)$ and $\mathbf{J}(\omega+\Delta \omega, z)$ lose coherence for sufficiently large values of $\Delta \omega$. In this case the products $\mathbf{J}(\omega, z)^{\dagger} \mathbf{J}\left(\omega_{3}, z\right)$ and $\mathbf{J}\left(\omega_{2}, z\right)^{\dagger} \mathbf{J}\left(\omega_{1}, z\right)$ in Eq. (2) are not necessarily equal to the identity matrix, causing the corresponding FWM products $\psi\left(\omega_{1}, \omega_{2}, \omega_{3}\right)$ to have dependencies on the exact PMD evolution along the fiber link.

From a nonlinear compensation perspective, we identify three relevant types of FWM contributions, as visualized in Fig. 1. By approximating the spectral autocorrelation function of PMD as a step function and defining $\mathbf{J}(\omega, z)$ and $\mathbf{J}(\omega+\Delta \omega, z)$ as statistically independent whenever $|\Delta \omega|$ is larger than some value $\Delta \omega_{\max }$ and $\mathbf{J}(\omega, z)=\mathbf{J}(\omega+\Delta \omega, z)$ otherwise, we find that:

(a) FWM products $\psi\left(\omega_{1}, \omega_{2}, \omega_{3}\right)$ satisfying $\mid \omega_{1}-$ $\omega_{2}|=| \omega_{3}-\omega \mid \leq \Delta \omega_{\max }$ are PMD-insensitive 
as they are always equal to

$$
\int_{0}^{L} \rho\left(\omega_{1}, \omega_{2}, \omega_{3}, z\right) \mathbf{a}\left(\omega_{3}\right) \mathbf{a}\left(\omega_{2}\right)^{\dagger} \mathbf{a}\left(\omega_{1}\right) \mathrm{d} z .
$$

(b) FWM products $\psi\left(\omega_{1}, \omega_{2}, \omega_{3}\right)$ that do not apply to above but satisfy $\left|\omega_{1}-\omega\right|=\mid \omega_{2}-$ $\omega_{3} \mid \leq \Delta \omega_{\max }$ are partially PMD-insensitive as their statistical average with respect to the polarization rotation matrices $\mathbf{J}(\omega, z), \mathbf{J}\left(\omega_{1}, z\right)$, $\mathbf{J}\left(\omega_{2}, z\right)$, and $\mathbf{J}\left(\omega_{3}, z\right)$ is equal to

$$
\frac{1}{2} \int_{0}^{L} \rho\left(\omega_{1}, \omega_{2}, \omega_{3}, z\right) \mathbf{a}\left(\omega_{1}\right) \mathbf{a}\left(\omega_{2}\right)^{\dagger} \mathbf{a}\left(\omega_{3}\right) \mathrm{d} z .
$$

Note that compared to the previous case, this term is halved and $\mathbf{a}\left(\omega_{1}\right), \mathbf{a}\left(\omega_{3}\right)$ are interchanged.

(c) FWM products $\psi\left(\omega_{1}, \omega_{2}, \omega_{3}\right)$ that do not apply to all of the above are PMD-sensitive. Their statistical average with respect to the polarization rotation matrices $\mathbf{J}(\omega, z), \mathbf{J}\left(\omega_{1}, z\right)$, $\mathbf{J}\left(\omega_{2}, z\right)$, and $\mathbf{J}\left(\omega_{3}, z\right)$ is always zero.

\section{Modified Voltera series DBP}

Conventional VS-DBP algorithms compensate for the accumulated nonlinear interference by following Eq. (1) and generating the various FWM terms using Eq. (2). However, since the PMD-evolution along the link is unknown, conventional algorithms ignore it by replacing the polarization rotation matrices $\mathbf{J}(\cdot, z)$ with the identity matrix in Eq. (2). Alternatively, our modified VS-DBP algorithm scans over all possible FWM contributions and accounts only for PMD-insensitive and partially PMD-insensitive contributions by estimating them using Eqs. (3) and (4), respectively. The value of $\Delta \omega_{\max }$, which defines the type of each FWM contribution, is a design parameter that needs to be optimized. Note that when $\Delta \omega_{\max }$ is equal to the backpropagated signal bandwidth, our modification reverts to the conventional VS-DBP algorithm. Figure 2 illustrates the different FWM contributions for a fixed $\omega$ as a function of $\omega_{1}$ and $\omega_{2}$. Conventional VS-DBP algorithms integrate over the entire domain $\omega_{1} \times \omega_{2}$ potentially degrading the performance due to the inclusion of mismatched PMDsensitive contributions corresponding to the orange area. Our modified algorithm ignores these contributions and accounts only for the green and blue areas, significantly alleviating the computational effort. Note that FWM contributions corresponding to the green area are fully compensated for as they always follow Eq. (3) under our assumptions. On the other hand, FWM contributions corresponding

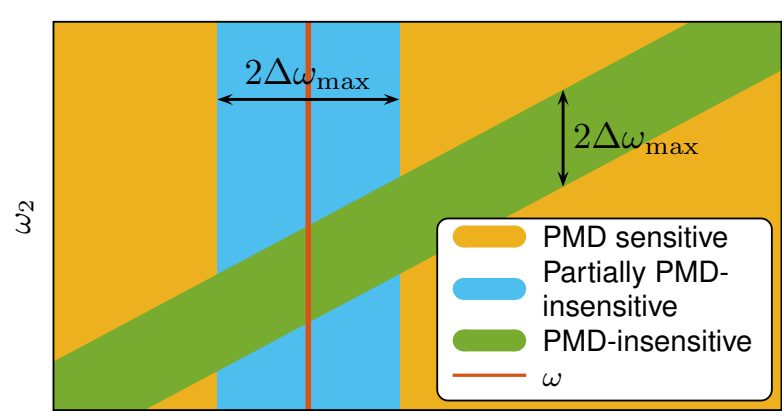

$\omega_{1}$

Fig. 2: Types of FWM contributions $\psi\left(\omega_{1}, \omega_{2}, \omega_{3}\right)$ for a fixed $\omega$ as a function of $\omega_{1}$ and $\omega_{2}$.

to the blue area are only compensated for their average value (with respect to PMD, see Eq. (4)); the difference between their average and exact realization is left uncompensated as it depends on the exact PMD evolution along the link. Coincidentally, the strongest FWM contributions for which $\int \rho\left(\omega_{1}, \omega_{2}, \omega_{3}, z\right) \mathrm{d} z$ is most significant are the terms located in the green and blue areas.

\section{Results and discussion}

We study through numerical simulations a pointto-point transmission system of $100-\mathrm{km}$ standard single-mode fiber (SSMF) spans with $\alpha=0.2$ $\mathrm{dB} / \mathrm{km}, \beta_{2}=17 \mathrm{ps} /(\mathrm{nm} \cdot \mathrm{km})$, and $\gamma=1.21 /(\mathrm{W} \cdot \mathrm{km})$. Each span is followed by one erbium-doped fiber amplifier with a noise figure of $4.5 \mathrm{~dB}$, compensating for the exact span loss. The transmitted signal consists of a single polarization-multiplexed 16-ary quadrature-amplitude modulated channel shaped using a square-root-raised cosine pulse with $\zeta=$ 0.01 roll-off factor. Forward propagation is simulated with SSFM steps of $0.1 \mathrm{~km}$ and static PMD is emulated at every step using a polarization scrambler, which uniformly ${ }^{8}$ scatters the state of polarization, and a retardation plate, which introduces a Gaussian distributed differential group delay. Seventy fiber realizations are simulated for each set of parameters so as to capture the stochastic nature of PMD. We perform VS-DBP at the receiver side on a per-span basis followed by an ideal matched filter, after which the signal-to-noise ratio (SNR) is estimated by comparing the transmitted and received symbols. We consider three PMD scenarios summarized in Tab. 1. For each PMD scenario we examine four different systems that result in the same normalized accumulated PMD (defined as $\Omega \tau_{c} \sqrt{L}$, where $\Omega$ and $\tau_{c}$ are the symbol rate and the fiber PMD coefficient, see Tab. 1).

Figure 3 shows the achieved SNR as a function of the input power for 100-GBaud transmission over a $1000-\mathrm{km}$ link with $0.5 \mathrm{ps} / \sqrt{\mathrm{km}}$ PMD coefficient.

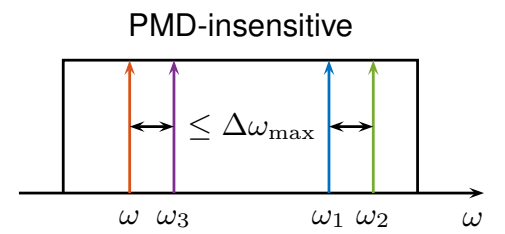

(a)
Partially PMD-insensitive

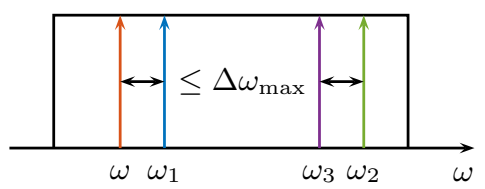

(b)

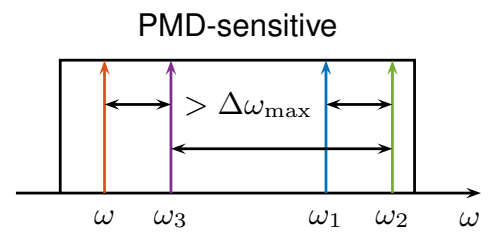

(c)

Fig. 1: Illustration of the three types of FWM contributions with respect to PMD. 


\begin{tabular}{|c|c|c|c|c|c|}
\hline \multirow{2}{*}{$\begin{array}{l}\text { Link } \\
\text { length } \\
L[\mathrm{~km}]\end{array}$} & \multirow{2}{*}{$\begin{array}{l}\text { Symbol } \\
\text { rate } \Omega \\
\text { [Gbaud] }\end{array}$} & \multicolumn{3}{|c|}{ PMD coeff. $\tau_{c}[\mathrm{ps} / \sqrt{\mathrm{km}}]$} & \multirow[b]{2}{*}{$\begin{array}{l}\text { Line } \\
\text { style }\end{array}$} \\
\hline & & $\begin{array}{l}\text { Weak } \\
\text { PMD }\end{array}$ & $\begin{array}{l}\text { Median } \\
\text { PMD }\end{array}$ & $\begin{array}{l}\text { Strong } \\
\text { PMD }\end{array}$ & \\
\hline & & 0.1 & 0.5 & 1 & \\
\hline 100 & 100 & 0.05 & 0.25 & 0.5 & --- \\
\hline 400 & 5( & 0.05 & 0.25 & 0.5 & $-\infty$ \\
\hline 4000 & 100 & 0.025 & 0.125 & 0.25 & $-\infty$. \\
\hline
\end{tabular}

Tab. 1: Transmission scenarios assumed in this work. The normalized accumulated PMD $\Omega \tau_{c} \sqrt{L}$ is equal to $0.158,0.791$, and 1.581 for all scenarios belonging to the weak, median, and strong PMD categories, respectively.

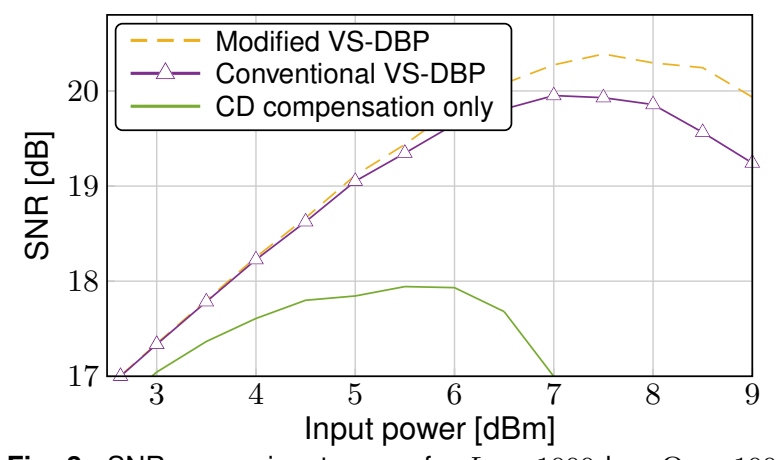

Fig. 3: SNR versus input power for $L=1000 \mathrm{~km}, \Omega=100$ Gbaud, $\tau_{c}=0.5 \mathrm{ps} / \sqrt{\mathrm{km}}$, and $\Delta \omega_{\max }=2 \pi \times 22 \mathrm{GHz}$.

Our VS-DBP algorithm with optimized $\Delta \omega_{\max }=$ $2 \pi \times 22 \mathrm{GHz}$ achieves $\sim 2.5 \mathrm{~dB}$ peak-SNR gain with respect to $\mathrm{CD}$ compensation only and $\sim 0.45$ dB SNR improvement with respect to conventional full-bandwidth VS-DBP.

Figure 4 shows the relative peak-SNR gains of our proposed method over the conventional fullbandwidth VS-DBP as a function of $\Delta \omega_{\max } / W$ (bottom $\mathrm{x}$-axis), where $W=2 \pi \times \Omega(1+\zeta)$ is the bandwidth of the signal, and the equivalent complexity reduction (calculated based on the ratio between the number of FWM terms considered in the modified and conventional VS-DBP, top $x$-axis). While full-bandwidth VS-DBP provides the highest gain for all cases with low PMD, it is significantly inferior to our modified VS-DBP algorithm in all cases with median and strong PMD. As the modified VS-DBP shifts from the conventional algorithm and $\Delta \omega_{\max }$ becomes smaller than the signal bandwidth, more PMD-sensitive contributions are ignored, yielding higher SNR gains and lower computational complexity. Maximum gains of up to $0.2 \mathrm{~dB}$ and $0.45 \mathrm{~dB}$ are achieved when $\Delta \omega_{\max }$ is about $40 \%$ and $20 \%$ of the overall signal bandwidth, corresponding to substantial complexity reductions of $15 \%$ and $42 \%$. The SNR gains then deteriorate as $\Delta \omega_{\max }$ gradually goes to zero and less FWM contributions are compensated for. We find that optimal performance is obtained when $\Delta \omega_{\max } \simeq$ $2.17 /\left(\tau_{c} \sqrt{L}\right)$, corresponding to the point where the spectral autocorrelation function ${ }^{9}$ of $\mathbf{J}(\omega, z)$ is 0.5 .

Figure 4 also shows that all four cases within each PMD scenario scale similarly with respect to the normalized $\Delta \omega_{\max }$, implying that one might use these results to predict the performance of the modified algorithm in other transmission scenar-

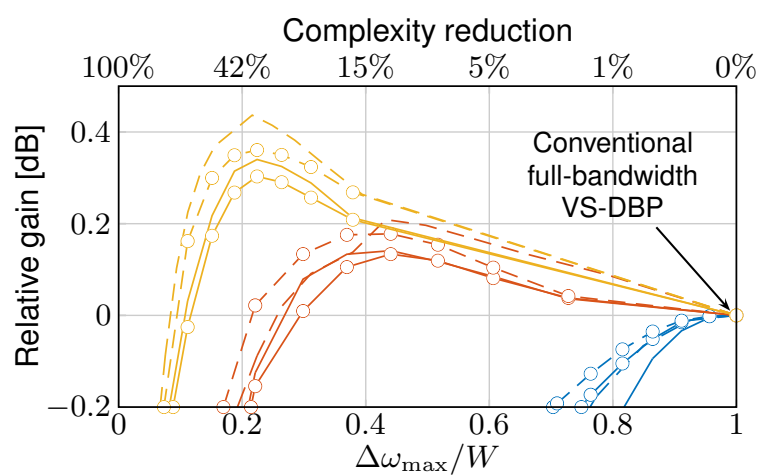

Fig. 4: Peak-SNR gain improvement vs. normalized $\Delta \omega_{\max }$ by the signal bandwidth $W$. The gains are measured with respect to the conventional full-bandwidth VS-DBP. See Tab. 1 for the lines legend.

ios. For example, joint backpropagation of $5 \times 100-$ Gbaud channels in a 4000-km system with a realistic PMD coefficient of $0.05 \mathrm{ps} / \sqrt{\mathrm{km}}$ is expected to fall under the category of strong PMD; in this case the gain and the complexity reduction of our algorithm are expected to be similar to what is shown by the yellow lines in Fig. 4 .

\section{Conclusions}

We demonstrated a modified VS-DBP algorithm that accounts for PMD by compensating for PMDinsensitive and partially PMD-insensitive nonlinear contributions only. This modification improves the achieved SNR and significantly reduces the required computational effort in the presence of PMD. We expect the acquired benefits of this method to be of great importance for future systems capable of performing large bandwidth joint multi-channel backpropagation where even small amounts of PMD can significantly limit the achievable gains.

\section{Acknowledgments}

We wish to thank P. Winzer for his invaluable feedback. This work was partially supported by the Swedish Research Council (VR) and the Ericsson's Research Foundation.

\section{References}

[1] R. J. Essiambre et al., "Electronic predistortion and fiber nonlinearity," PTL, 18(17), p. 1804, 2006.

[2] W. Yan et al., "Low complexity digital perturbation backpropagation," ECOC, Tu.3.A.2., 2011.

[3] F. P. Guiomar et al., "Mitigation of intra-channel nonlinearities using a frequency-domain Volterra series equalizer," Opt. Express, 20(2), p. 1360, 2012.

[4] K. V. Peddanarappagari and M. Brandt-Pearce, "Volterra series transfer function of single-mode fibers," JLT, 15(12), p. 2232, 1997.

[5] F. Yaman and G. Li, "Nonlinear impairment compensation for polarization-division multiplexed WDM transmission using digital backward propagation," Photon. J., 2(5), p. 816, 2010.

[6] C. B. Czegledi et al., "Digital backpropagation accounting for polarization-mode dispersion," Opt. Express, 25(3), p. 1903, 2017.

[7] K. Goroshko et al., "Overcoming performance limitations of digital back propagation due to polarization mode dispersion," ICTON, Mo.B1.4, 2016.

[8] C. B. Czegledi et al., "Polarization drift channel model for coherent fibre-optic systems," Sci. Rep., 6(21217), 2016.

[9] C. B. Czegledi et al., "Temporal stochastic channel model for absolute polarization state and polarization-mode dispersion," OFC, Th3F.2, 2017. 